\title{
A novel method for sentinel lymph node mapping using magnetite in patients with non-small cell lung cancer
}

Taku Nakagawa, MDa

Yoshihiro Minamiya, MD $^{\mathrm{a}}$

Yoshihisa Katayose, MD

Hajime Saito, MD

Kousei Taguchi, MD

Hiroshi Imano, MD

Hitoshi Watanabe ${ }^{\mathrm{b}}$

Katsuhiko Enomoto, $M D^{\mathrm{b}}$

Masato Sageshima, $\mathrm{MD}^{\mathrm{c}}$

Tomoaki Ueda, $\mathrm{PhD}^{\mathrm{d}}$

Jun-ichi Ogawa, MDa

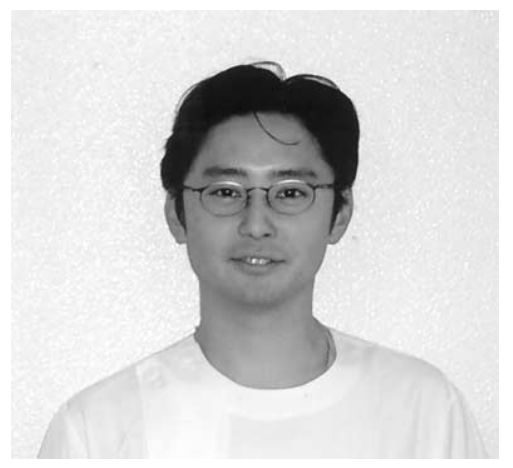

Dr Nakagawa

From the Second Department of Surgery ${ }^{\mathrm{a}}$ and First Department of Pathology, ${ }^{\text {b }}$ Akita University School of Medicine; Division of Clinical Pathology, ${ }^{\mathrm{c}}$ Akita University Hospital, Akita City, Japan; and Kansai Research Institute, ${ }^{\mathrm{d}}$ Kyoto Research Park 17, Kyoto, Japan.

This work was partially supported by a Grant-in-Aid for Scientific Research from the Ministry of Education, Culture, Sports, Science and Technology of Japan.

Received for publication Sept 30, 2002; revisions requested Nov 25, 2002; revisions received Dec 9, 2002; accepted for publication Jan 9, 2003.

Address for reprints: Yoshihiro Minamiya, MD, Associate Professor of Thoracic Surgery, Second Department of Surgery, Akita University School of Medicine, 1-1-1 Hondo, Akita City 010-8543, Japan (Email: minamiya@med.akita-u.ac.jp).

J Thorac Cardiovasc Surg 2003;126:563-7

Copyright (@) 2003 by The American Association for Thoracic Surgery

$0022-5223 / 2003 \$ 30.00+0$

doi:10.1016/S0022-5223(03)00216-2
Objective: The detection rate of sentinel lymph nodes in patients with non-small cell lung cancer using isosulfan blue dye is too low for clinical use. Although exposure to radioactivity is reportedly minimal, special procedures are nonetheless required when a radioactive isotope is used as a tracer. Therefore, to eliminate the need for a radioactive tracer and to obtain a better detection rate than is obtained with isosulfan blue dye, we have developed a novel method that employs magnetite as the tracer. The aim of the present study was to test the feasibility of this technique.

Methods: The tracer employed was ferumoxides, a colloidal superparamagnetic iron oxide of nonstoichiometric magnetite. Thirty-eight non-small cell lung cancer patients participated in the study; each received $5 \mathrm{~mL}$ of ferumoxides, injected around the tumor intraoperatively. Fifteen minutes after injection, lung resection and lymph node dissection were carried out. The magnetic force within the lymph nodes was measured using a highly sensitive handheld magnetometer ex vivo. All lymph nodes were also subjected to conventional histological analysis.

Results: The rate of detection of sentinel lymph nodes was $81.6 \%$ (31/38). The accuracy, sensitivity, and false-negative rates were $96.8 \%$ (30/31), $85.7 \%$ (6/7), and $14.3 \%(1 / 7)$, respectively.

Conclusion: Intraoperative sentinel lymph node mapping using ferumoxides and a highly sensitive magnetometer is a safe, accurate, and sensitive way to detect sentinel lymph nodes in non-small cell lung cancer patients.

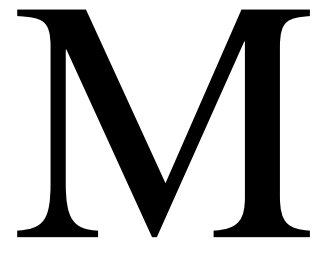

ediastinal lymph node dissection (MLND) is an effective therapeutic procedure when carried out in patients with metastatic non-small cell lung cancer (NSCLC); moreover, the status of lymph node metastasis is an important prognostic indicator. ${ }^{1,2}$ On the other hand, MLND is not therapeutic and may even be harmful for patients without lymph node metastasis. 


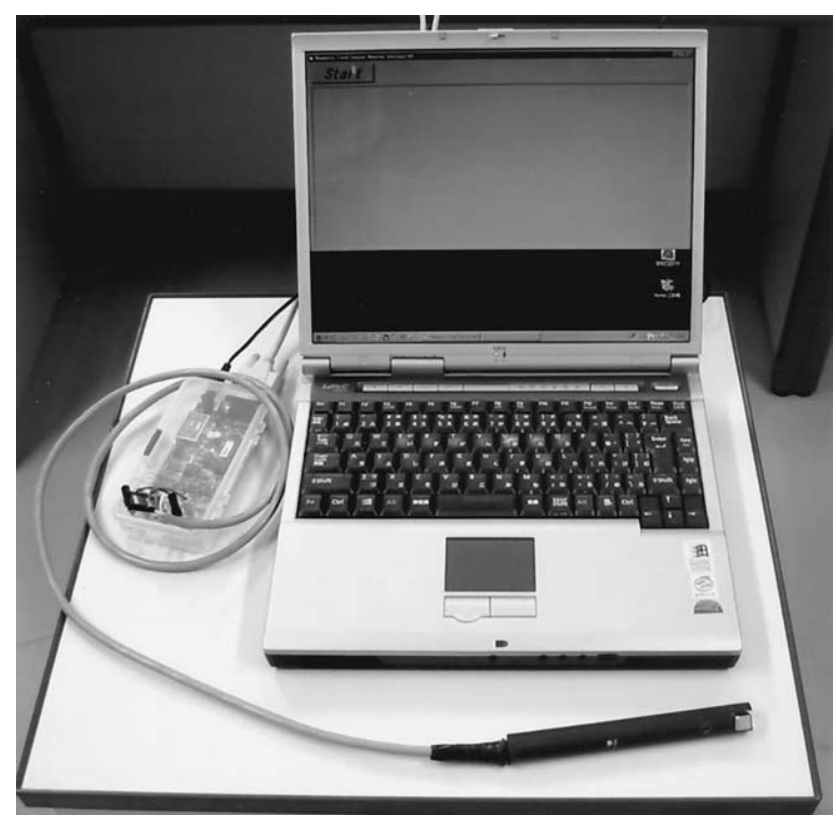

Figure 1. The handheld magnetometer.

The sentinel lymph node (SLN) concept is that lymphatic flux from a primary tumor initially flows into an SLN. If this concept is correct, then when metastasis is not found in an SLN, it most likely will not be present in more distal lymph nodes. Although the benefit of SLN mapping and biopsy remains controversial, it has gained acceptance in recent years as a way to avoid the complications of lymph node dissection and has become a common procedure in breast cancer and melanoma. ${ }^{3,4}$ On the other hand, the SLN concept is still under investigation and not yet approved for NSCLC.

Thus far, there have been only 5 reports from 4 groups, including this paper, on the use of SLN mapping with NSCLC. ${ }^{5-8}$ Two tracers, isotope and isosulfan blue dye, have been used to locate SLNs intraoperatively. ${ }^{5-8}$ Little and colleagues $^{8}$ injected peritumoral regions with isosulfan blue dye; Liptay and coworkers ${ }^{6,7}$ intraoperatively injected technetium $99 \mathrm{~m}$ sulfur colloid directly into lung tumors and detected SLNs with a gamma probe; and Nomori's group ${ }^{5}$ injected technetium $99 \mathrm{~m}$ tin colloid into peritumoral regions preoperatively using a computed tomography-guided technique, and then detected SLNs intraoperatively using a gamma probe. These 3 groups demonstrated the feasibility of their methods and the utility of the SLN concept in NSCLC. Unfortunately, none of these techniques is problem-free: precautions must be taken to minimize exposure to radioisotopes; it is difficult to recognize isosulfan blue dye in thoracic lymph nodes because of anthoracosis; and in Japan the use of radioisotopes is strictly prohibited except in designated areas.
To solve these problems, we developed a new method for detecting SLN using magnetic force. As a tracer, we applied colloidal ferumoxides, which is a superparamagnetic iron oxide of nonstoichiometric magnetite that has already been approved for clinical use in numerous countries as a contrast material for magnetic resonance imaging. We also developed a highly sensitive, handheld magnetometer to detect the magnetic force of the ferumoxides within SLNs. Using this technique, we were able to detect SLNs safely in the patients with NSCLC, without the use of radioisotopes or dye.

\section{Patients and Methods}

\section{Tracer}

Ferumoxides, a colloidal superparamagnetic iron oxide of nonstoichiometric magnetite (Feridex; Eiken Chemistry Co, Tokyo, Japan), was used as the tracer. Particle diameters ranged from 70 to $140 \mathrm{~nm}$; the average size was about $100 \mathrm{~nm}$.

\section{Patients}

Thirty-eight consecutive patients with NSCLC were enrolled in the study between May 2001 and February 2002 after obtaining signed, informed consent. After the preoperative evaluation, the patients were taken to an operating room, and the standard preparations were made for a thoracotomy and lung resection. None of the patients received preoperative chemotherapy or radiotherapy.

\section{Intraoperative Technique}

After thoracotomy, ferumoxides was injected around the tumor; a total dosage of $5.0 \mathrm{~mL}$ was administered in four $1.25-\mathrm{mL}$ doses in a 4-quadrant region. To avoid surgical destruction of the lymphatic system of the pleura and along the bronchi and vessels, we waited for 15 minutes after injection before proceeding. To promote migration of ferumoxides throughout the lymphatic vessels, ventilation was continued during the waiting period. The time from the injection of the ferumoxides to the harvest of the individual lymph nodes was recorded. The lymph node station was based on the classification by Naruke and colleagues. ${ }^{9}$ After the operation, the magnetic force in the lymph nodes was measured ex vivo using a highly sensitive, handheld magnetometer developed in our institute (Figure 1). This device enabled us to measure magnetic forces of up to $3 \mu \mathrm{T}$ in the operating room (patent pending). Magnetic force values were expressed in terms of millivolts because the absolute magnetic force varied depending on the distance between the magnetometer and the samples. Ferumoxides migration was considered successful if a specific nodal station counts was greater than $5.0 \mathrm{mV}$; the background signal was $3.0 \mathrm{mV}$. If a lymph node station was found to have the highest or second highest signal and ex vivo measurements were greater than $5.0 \mathrm{mV}$, that station was classified to be an SLN.

\section{Pathologic Evaluation}

All dissected lymph nodes were sectioned and conventionally examined using hematoxylin and eosin staining. 


\section{Results}

The patient characteristics are summarized in Table 1. Pathological analysis revealed that 31 of the 38 patients had no nodal metastasis. No complications related to the injection with ferumoxides were detected, and successful migration was detected in 31 of the 38 patients (detection rate $81.6 \%$ ). The mean ferumoxides migration time was 85.7 minutes. To confirm migration to the lymph nodes, we histologically examined a magnetic force-positive lymph node and found that ferumoxides was present mainly in the macrophages (Figure 2).

Table 2 summarizes the results of SLN identification. Histological analysis revealed lymph node metastases in 7 of the 31 patients ( 24 of the 31 patients were N0); metastases were detected in 6 of the 31 patients with ferumoxidespositive lymph nodes. Of those 6 patients, 5 showed metastases in both ferumoxides-positive and ferumoxidesnegative nodes; in these cases, it was unclear which node was affected first or whether the ferumoxides-positive node had served as an SLN. However, it was notable that the remaining 1 patient showed metastasis exclusively in the ferumoxides-positive node, suggesting that indeed the ferumoxides-positive node was the SLN and that the SLN concept is applicable to NSCLC. Thus, we were able to accurately identify the SLN in 30 of 31 patients $(96.8 \%)$, and we inaccurately identified the SLN in only 1 patient. The sensitivity and false-negative rates were $85.7 \%$ and $14.3 \%$, respectively.

The locations of the SLNs detected by our method are shown in Table 3 . In 8 of the 31 patients (25.8\%), the SLN was located in the $\mathrm{N} 2$ station alone, which suggests that the first station of lymphatic drainage was the mediastinal lymph nodes. These were 5 subcarinal level 7 nodes from 4 lower lobe tumors and 1 right upper lobe tumor; 2 paratracheal level 4 nodes from right lower lobe and left upper lobe tumors; 1 upper paratracheal level 2 node from a right middle lobe tumor; and 1 pulmonary ligament level 9 node from a right lower lobe tumor. In 1 of those 8 patients, SLNs were located in both levels 4 and 9 from a right lower lobe tumor.

\section{Discussion}

In the present study, we used a new ex vivo technique in which ferumoxides was detected with a highly sensitive, handheld magnetometer in patients with NSCLC. The detection, accuracy, sensitivity, and false-negative rates obtained with this method were $81.6 \%, 96.8 \%, 85.7 \%$, and $14.3 \%$, respectively. We therefore believe this method to be a safe and effective way to detect SLNs in NSCLC patients.

The primary utility of SLN mapping and biopsy is to avoid nontherapeutic lymph node dissection and the complications it causes. Indeed, SLN mapping and biopsy were developed as techniques for staging the lymphatic basin without the potential morbidity of lymphedema and nerve
TABLE 1. Patient characteristics

\begin{tabular}{|c|c|c|c|}
\hline Patients & 38 & Tumor location & \\
\hline Age (y) & & RUL & 8 \\
\hline Mean & $65.4 \pm 9.7$ & RML & 3 \\
\hline Range & $42-79$ & RLL & 8 \\
\hline Sex & $\begin{array}{l}\text { Female } 14, \\
\text { Male } 24\end{array}$ & LUL & 11 \\
\hline & & LLL & 8 \\
\hline \multicolumn{4}{|l|}{$\begin{array}{l}\text { Pathological nodal } \\
\text { status }\end{array}$} \\
\hline NO & 31 & Tumor size $(\mathrm{cm})$ & \\
\hline N1 & 4 & Mean & $2.9 \pm 1.7$ \\
\hline N2 & 3 & Range & $0.8-8.0$ \\
\hline Pathological stage & & Histological type & \\
\hline Stage IA & 16 & ad & 27 \\
\hline Stage IB & 12 & $\mathrm{sq}$ & 9 \\
\hline Stage IIA & 1 & $\mathrm{La}$ & 2 \\
\hline Stage IIB & 4 & Operation & \\
\hline Stage IIIA & 2 & Lobectomy & 33 \\
\hline Stage IIIB & 2 & $\begin{array}{l}\text { Sleeve } \\
\quad \text { lobectomy }\end{array}$ & 2 \\
\hline Stage IV & 1 & Pneumonectomy & 3 \\
\hline
\end{tabular}

$R U L$, Right upper lobe; $R M L$, right middle lobe; $R L L$, right lower lobe; $L U L$, left upper lobe; $L L L$, left lower lobe; $a d$, adenocarcinoma; $s q$, squamous cell carcinoma; $L a$, large cell carcinoma.

injury in cases of melanoma ${ }^{3}$ or lymphedema of the arm in cases of breast cancer. ${ }^{4}$ Lung resection for NSCLC with MLND leads to greater production of postoperative exudate than lung resection without MLND, although the morbidity of MLND is not excessive, ${ }^{10}$ and the procedure is therapeutic. ${ }^{1}$ Liptay and colleagues ${ }^{6,7}$ argued that the primary function of SLN mapping and biopsy is to direct pathologic examination to specific SLNs, after which more sensitive techniques can be employed on a limited amount of tissue to detect occult micrometastatic disease. ${ }^{6,7}$ Nomori and his colleagues $^{5}$ emphasized the advantage of avoiding nontherapeutic lymph node dissection, especially in segmentectomy for the early-stage NSCLC. We believe that we can also apply SLN mapping and biopsy to video-assisted thoracoscopic surgery (VATS) for NSCLC. Although lymph node dissection with VATS lobectomy is technically feasible, with remnants of only $2 \%$ to $3 \%$ of lymph node and other tissues, ${ }^{11}$ it remains technically difficult to dissect mediastinal lymph nodes completely, particularly level 4 in left lung cancer. If we could apply SLN mapping and biopsy to VATS lobectomy for NSCLC, we could avoid nontherapeutic and technically difficult MLND.

Although radioactive isotopes or isosulfan blue dye are currently used to locate SLNs in breast cancer, ${ }^{12,13}$ neither of these techniques is problem-free. Precautions are needed to minimize exposure to radioisotopes, and anaphylactic reactions to isosulfan blue have been reported. ${ }^{14}$ Furthermore, it is difficult to recognize isosulfan blue dye in thoracic lymph nodes because of the anthoracosis. In fact, the detection rate of SLNs in patients with NSCLC using isosulfan blue dye is quite low. ${ }^{8}$ 


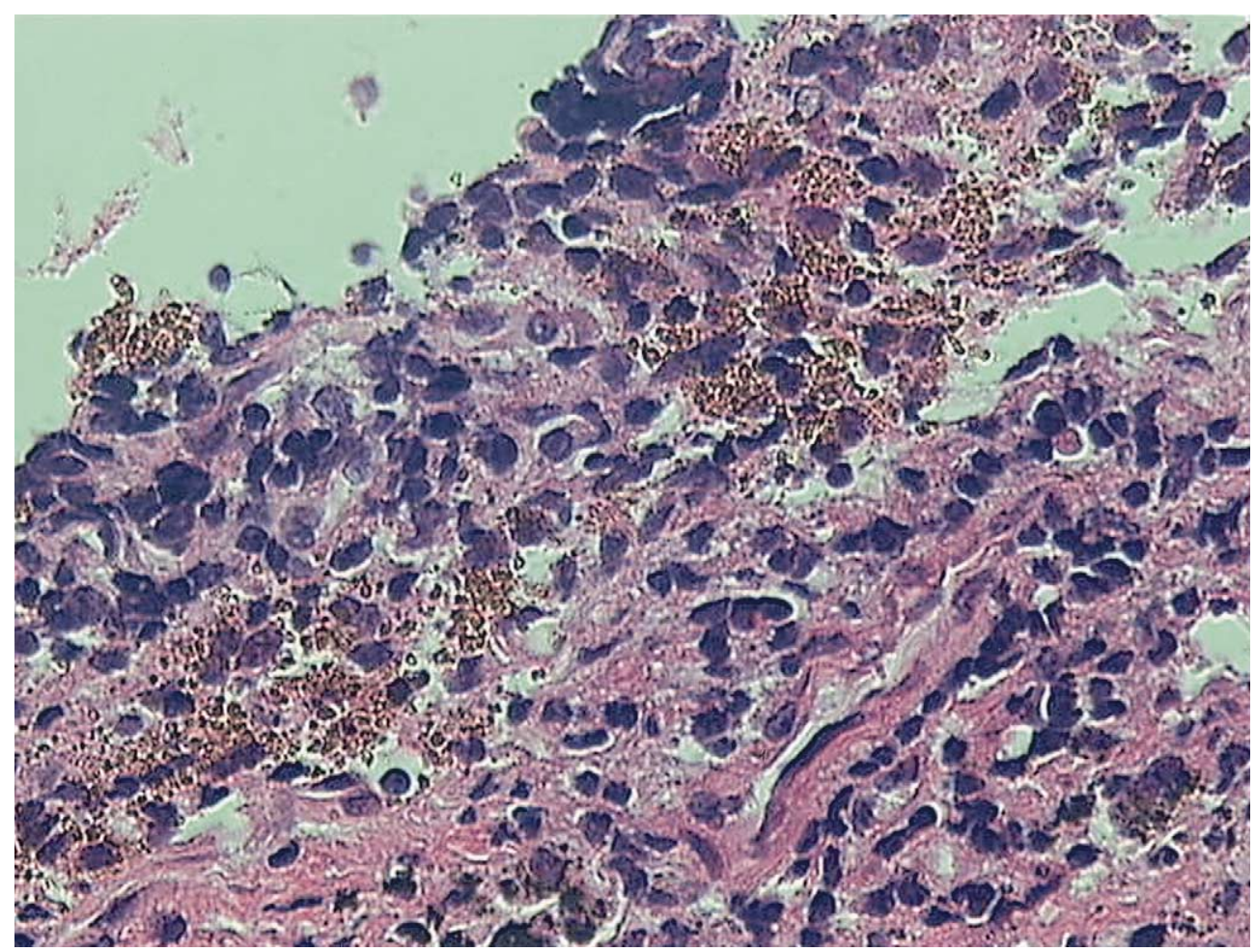

Figure 2. Sentinel lymph node detected with magnetic force. Representative hematoxylin and eosin-stained micrograph $(40 \times)$ confirming ferumoxides migration to the lymph node detected with handheld magnetometer. Ferumoxides was mainly observed in macrophages within the harvested lymph nodes.

\section{TABLE 2. Sentinel lymph node identification}

\begin{tabular}{|c|c|c|}
\hline Identification & $\mathbf{n}$ & Migration time \\
\hline $\begin{array}{l}\text { SLN identified (successful } \\
\text { migration of FE) }\end{array}$ & 31 of $38(81.6 \%)$ & $85.7 \min (22-172)$ \\
\hline Number of metastases & 7 of $31(22.6 \%)$ & \\
\hline $\begin{array}{l}\text { Metastases in FE }(+) \\
\text { nodes }\end{array}$ & 6 of $31(19.4 \%)$ & \\
\hline $\begin{array}{l}\text { Metastases in FE }(-) \\
\text { nodes (inaccurately } \\
\text { identified SLN) }\end{array}$ & 1 of $31(3.2 \%)$ & \\
\hline Accurately identified SLN & 30 of $31(96.8 \%)$ & \\
\hline $\begin{array}{l}\text { Sensitivity (metastases in } \\
\mathrm{FE}(+) \text { nodes/number of } \\
\text { metastases) }\end{array}$ & 6 of $7(85.7 \%)$ & \\
\hline $\begin{array}{l}\text { False-negative (metastases } \\
\text { in FE }(-) \text { nodes/number } \\
\text { of metastases) }\end{array}$ & 1 of $7(14.3 \%)$ & \\
\hline \multicolumn{3}{|l|}{ SLN not identified } \\
\hline No migration of FE & 7 of $38(18.4 \%)$ & \\
\hline
\end{tabular}

In the breast cancer field, numerous investigators have reported that when using a radioactive tracer, the radiation risk to surgeons, pathologists, and other medical staff to
TABLE 3. Sentinel lymph node location

\begin{tabular}{lcccc}
\hline $\begin{array}{l}\text { Tumor } \\
\text { location }\end{array}$ & $\begin{array}{c}\text { Number of } \\
\text { cases }\end{array}$ & $\begin{array}{c}\text { N1 } \\
\text { alone }\end{array}$ & $\begin{array}{c}\text { N2 } \\
\text { alone }\end{array}$ & $\begin{array}{c}\text { N1 and } \\
\text { N2 }\end{array}$ \\
\hline RUL & 6 & 2 & 1 & 3 \\
RML & 3 & 1 & 1 & 1 \\
RLL & 6 & 2 & 3 & 1 \\
LUL & 9 & 8 & 1 & 0 \\
LLL & 7 & 2 & 2 & 3 \\
Total & 31 & 15 & 8 & 8 \\
\hline
\end{tabular}

$R U L$, Right upper lobe; $R M L$, right middle lobe; $R L L$, right lower lobe; $L U L$, left upper lobe; $L L L$, left lower lobe.

radiation is minimal, ${ }^{15-17}$ and the risk to patients is no greater than with numerous other medical procedures. ${ }^{16}$ The radiation levels in the waste are also low. ${ }^{15}$ Nevertheless, radioactive materials always need to be handled with care to avoid secondary contamination; for example, Waddington and coworkers ${ }^{16}$ reported that surgical swabs can contain up to $22 \%$ of the administered activity. Awareness of and adherence to radiation safety regulations are strongly recommended to minimize exposure and to safely apply the technique. ${ }^{17}$ Thus, although the radio-guided method is quite a safe procedure, special procedures must be employed. Furthermore, the use of radioisotopes in Japan is 
strictly limited to designated areas. By contrast, no such precautions are required when handling magnetite. We therefore believe that magnetic-guided SLN mapping is more practical and effective than radio-guided mapping.

The SLN detection rate of $81.6 \%$ obtained in our study compares favorably with the $82 \%$ rate reported by Liptay and colleagues ${ }^{6}$ for radio-guided mapping using technetium $99 \mathrm{~m}$ sulfur colloid and the $87 \%$ rate reported by Nomori and coworkers ${ }^{5}$ for radio-guided mapping using technetium $99 \mathrm{~m}$ tin colloid. Notably, Nomori's group also reported that patients whose sentinel nodes could not be identified had a significantly lower ratio of forced expiratory volume in 1 second to forced vital capacity than did patients with identifiable sentinel node. ${ }^{5}$ Destruction of the lymphatic vessels in the alveolar septa and along the airway in chronic obstructive pulmonary disease may explain this phenomenon, though full clarification of the reason for the failure to detect SLNs in some cases awaits further investigation.

The particle diameters of technetium 99m sulfur colloid, ferumoxides, and technetium $99 \mathrm{~m}$ tin colloid are approximately $40 \mathrm{~nm}, 100 \mathrm{~nm}$, and $1000 \mathrm{~nm}$, respectively. More time was required for ferumoxides to migrate to a sentinel node station ( 85.7 minutes) than for technetium $99 \mathrm{~m}$ sulfur colloid (10 to 15 minutes). ${ }^{6}$ By contrast, because of its comparatively large size, the migration time for technetium $99 \mathrm{~m}$ tin colloid was at least 6 hours, which meant that it had to be injected preoperatively. ${ }^{5}$ On the other hand, ferumoxides can be injected intraoperatively, which is advantageous as it enables us to avoid the risks of pneumothorax, bleeding, and tumor seeding. Moreover, it eliminates the need of another preoperative procedure. A disadvantage of the smaller particles is the risk of false-positives, as small particles sometimes pass through the true SLN and flow up the chain of nodes. In fact, we detected magnetic force in non-SLNs but were able to distinguish the SLN from other nodes based on the intensity of the signal.

In the present study, the SLN was mediastinal (N2) in 8 of 31 patients $(25.8 \%)$, which is consistent with previous reports. ${ }^{5-7}$ The prognosis of patients with skip N2 metastasis is reportedly better than patients with other N2 metastases and similar to those with $\mathrm{N} 1$ metastasis. ${ }^{18}$ The incidence of skip metastasis in mediastinal lymph nodes is between $20 \%$ and $40 \%$ in NSCLC. ${ }^{19-21}$ Perhaps SLN investigation will shed light on the mechanism of skip metastasis in NSCLC.

In summary, we have developed a new ex vivo method of SLN mapping using ferumoxides with a highly sensitive handheld magnetometer. This method appears to be as accurate and sensitive as SLN mapping using technetium; however, only a limited number of patients were investigated. Further investigation will thus be required to confirm the feasibility and safety of this approach. In addition, our magnetometer is not yet suitable for in vivo SLN mapping; development of such an instrument is planned, however.
We thank Ms Mitsuko Sato and Jun Kodama for secretarial support.

\section{References}

1. Keller SM, Adak S, Wagner H, Johnson DH. Mediastinal lymph node dissection improves survival in patients with stages II and IIIa nonsmall cell lung cancer. Eastern Cooperative Oncology Group. Ann Thorac Surg. 2000;70:358-65.

2. Naruke T, Goya T, Tsuchiya R, Suemasu K. The importance of surgery to non-small cell carcinoma of lung with mediastinal lymph node metastasis. Ann Thorac Surg. 1988;46:603-10.

3. Shen J, Wallace AM, Bouvet M. The role of sentinel lymph node biopsy for melanoma. Semin Oncol. 2002;29:341-52.

4. Petrek JA, Senie RT, Peters M, Rosen PP. Lymphedema in a cohort of breast carcinoma survivors 20 years after diagnosis. Cancer. 2001;92: 1368-77.

5. Nomori H, Horio H, Naruke T, Orikasa H, Yamazaki K, Suemasu K. Use of technetium-99m tin colloid for sentinel lymph node identification in non-small cell lung cancer. J Thorac Cardiovasc Surg. 2002; 124:486-92.

6. Liptay MJ, Grondin SC, Fry WA, et al. Intraoperative sentinel lymph node mapping in non-small-cell lung cancer improves detection of micrometastases. J Clin Oncol. 2002;20:1984-8.

7. Liptay MJ, Masters GA, Winchester DJ, et al. Intraoperative radioisotope sentinel lymph node mapping in non-small cell lung cancer. Ann Thorac Surg. 2000;70:383-90.

8. Little AG, DeHoyos A, Kirgan DM, Arcomano TR, Murray KD. Intraoperative lymphatic mapping for non-small cell lung cancer: the sentinel node technique. J Thorac Cardiovasc Surg. 1999;117:220-34.

9. Naruke T, Suemasu K, Ishikawa S. Lymph node mapping and curability at various levels of metastasis in resected lung cancer. $J$ Thorac Cardiovasc Surg. 1978;76:832-9.

10. Bollen EC, van Duin CJ, Theunissen PH, vt Hof-Grootenboer BE, Blijham GH. Mediastinal lymph node dissection in resected lung cancer: morbidity and accuracy of staging. Ann Thorac Surg. 1993;55:961-6.

11. Sagawa M, Sato M, Sakurada A, et al. A prospective trial of systematic nodal dissection for lung cancer by video-assisted thoracic surgery: can it be perfect? Ann Thorac Surg. 2002;73:900-4.

12. Krag DN, Weaver DL, Alex JC, Fairbank JT. Surgical resection and radiolocalization of the sentinel lymph node in breast cancer using a gamma probe. Surg Oncol. 1993;2:335-9.

13. Giuliano AE, Kirgan DM, Guenther JM, Morton DL. Lymphatic mapping and sentinel lymphadenectomy for breast cancer. Ann Surg. 1994;220:391-401.

14. Albo D, Wayne JD, Hunt KK, et al. Anaphylactic reactions to isosulfan blue dye during sentinel lymph node biopsy for breast cancer. Am J Surg. 2001;182:393-8.

15. Motta C, Turra A, Farina B, Ostan A, Ramella S, Cartia GL. Radioguided surgery of breast cancer: radiation protection survey. Tumori. 2000;86:372-4.

16. Waddington WA, Keshtgar MR, Taylor I, Lakhani SR, Short MD, Ell PJ. Radiation safety of the sentinel lymph node technique in breast cancer. Eur J Nucl Med. 2000;27:377-91.

17. Miner TJ, Shriver CD, Flicek PR, et al. Guidelines for the safe use of radioactive materials during localization and resection of the sentinel lymph node. Ann Surg Oncol. 1999;6:75-82.

18. Andre F, Grunenwald D, Pignon JP, et al. Survival of patients with resected N2 non-small-cell lung cancer: evidence for a subclassification and implications. J Clin Oncol. 2000;18:2981-9.

19. Asamura H, Nakayama H, Kondo H, Tsuchiya R, Naruke T. Lobespecific extent of systematic lymph node dissection for non-small cell lung carcinomas according to a retrospective study of metastasis and prognosis. J Thorac Cardiovasc Surg. 1999;117:1102-11.

20. Okada M, Tsubota N, Yoshimura M, Miyamoto Y, Matsuoka H. Prognosis of completely resected pN2 non-small cell lung carcinomas: what is the significant node that affects survival? J Thorac Cardiovasc Surg. 1999;118:270-5.

21. Riquet M, Hidden G, Debesse B. Direct lymphatic drainage of lung segments to the mediastinal nodes. An anatomic study on 260 adults. J Thorac Cardiovasc Surg. 1989;97:623-32. 